\title{
TRATAMIENTO DEL DUELO: EXPLORACIÓN Y PERSPECTIVAS
}

\author{
TREATMENT OF GRIEF: EXPLORATION AND PERSPECTIVES
}

Verónica Romero

Asociación Española Contra el Cáncer, Junta Provincial de Granada

Resumen

El duelo es la reacción emocional que se da ante una pérdida. Estas reacciones son universales, afectan a las diferentes dimensiones de la persona y suelen expresarse de forma diversa según la cultura en donde se desenvuelven. La complicación del proceso del duelo, conlleva unas series de manifestaciones clínicas que vulneran y comprometen la salud física y emocional de las personas, y por ello, requieren ser atendidas por profesionales especialistas que puedan aliviar su sufrimiento consecuente. En las últimas décadas se ha invertido mucho trabajo en la descripción de cuáles son los criterios que nos ayuden a diferenciar los duelos normativos de las complicaciones del mismo. Se ha propuesto, tras las evidencias encontradas en las investigaciones realizadas, una entidad clínica propia de los cuadros más complicados, diferenciada de otros trastornos psicológicos. A partir de aquí se podría hablar en un lenguaje común a la hora de definirlo y evaluarlo, facilitando el desarrollo de estudios y resultados aplicables posteriormente al ámbito clínico. En referencia al tratamiento del duelo, en la literatura científica se despliegan un gran número de investigaciones que abordan esta problemática desde las diferentes orientaciones psicológicas, desde donde se sustentan sus procedimientos y ofrecen direcciones y líneas de intervención que ayuden a poder consolar la magnitud de esta vivencia. A pesar de ello, las diferentes revisiones que

\section{Abstract}

The mourning is the emotional reaction because of a death. These reactions are universals; affect at the different dimensions of the individual and usually to be expressed in a diverse way depends on the culture where it is developed. The complication of the process of the mourning entails a series of clinic manifestations that damage and endanger the physic and emotional health of the people and thus, require to be attended for specialist professionals that can give relief his consequent suffering. In the last decades have been inverted a lot of energies on the description for which criterions help us to differentiate the normative mourning than the difficulties of itself. It has been proposed, after the evidences found in the investigations realized, a clinic entity typical from the symptoms more complex, diversified of other psychological disorders. From here it could be spoken in a communal language to define and evaluate it, facilitating the development of studies and outcomes applicable subsequent to the clinic field. According to the treatment of the mourning, in the scientific literature displays a big number of studies that address this problematic from the different psychological orientations, from where support his procedures and offer directions and lines of intervention helping to give comfort the magnitude of this experience. However, the different revisions realized to value the

\section{Correspondencia:}

Verónica Romero

Asociación Española Contra el Cáncer, Junta Provincial de Granada

C) Tortola, $n^{\circ} 3$ (bajo)

18014-Granada

E-mail: passduchat@hotmail.com 
se han realizado para valorar las respuestas de las estrategias y técnicas utilizadas, no han mostrado resultados concluyentes, hacia qué tipo de intervención pudiera ser más eficaz. En este artículo se recopilan los hallazgos recientes que se han desarrollado en este ámbito y ofrece un abordaje de los procedimientos y estudios que muestran buenos resultados.

Palabras clave: Duelo complicado, intervención, revisiones, tratamientos eficaces. answers of the strategies and techniques have been used, have not showed concluding results through which type of intervention could be more effective. This article compiles the recent discoveries have been developed in this sphere and offers an approach of the procedures and studies which show the best results.

Key words: Complicated grief, intervention, revision, effectives treatments.

los años setenta, cuando empezaron a surgir una serie de estudios encaminados a la búsqueda de criterios diagnósticos que operativizaran una entidad clínica propia, diferenciada de los demás trastornos psicológicos.

Prigerson, ha sido una de los autores que ha liderado un amplio estudio dirigido a la validación de estos criterios. Como una de sus aportaciones más relevantes destaca, la elaboración del Inventario de Duelo Complicado (IDC) (3), que ha sido ampliamente utilizado como herramienta de evaluación. Su trabajo ha ido dirigido hacia la descripción y conocimiento de los criterios de riesgo que pueden presentarse, al estudio de su naturaleza y etiología, así como de las consecuencias físicas y emocionales que pueden desencadenar la complicación del proceso de duelo ${ }^{(4)}$. Asimismo, realiza una valoración de las diferentes líneas de intervención y tratamientos del duelo complicado, proponiendo como terapias con respuestas más alentadoras las establecidas bajo la corriente psicodinámica y la psicoterapia con orientación cognitivo-conductual. Prigerson y su grupo de trabajo, enfatizaban además que los estudios que incluían a participantes con criterios altos de riesgo de "dolor traumáti$\mathrm{CO}^{\prime \prime}$ en personas en duelo, se beneficiaban más de la efectividad de esos tratamientos. A pesar de ello, plantean la necesidad del desarrollo de terapias específicas para este tipo de dolor, y de estudios controlados 
que evidencien cuáles son las terapias más beneficiosos para estos pacientes ${ }^{(5)}$.

En el año 2009, se realizó una investigación para consensuar los criterios diagnósticos del duelo complicado ${ }^{(4)}$. Se puso en marcha un estudio prospectivo, en el que se evaluaron a 317 participantes en diferentes momentos temporales. A partir de aquí, los autores defienden una entidad propia que describa la idiosincrasia del proceso, que a su vez sea diferenciada de las manifestaciones que pudiera compartir y que están presentes con el trastorno depresivo mayor, el trastorno de estrés postraumático y el trastorno de ansiedad generalizada $^{(6)}$. Describen el duelo complicado como una combinación de síntomas de ansiedad de separación y de emociones, cogniciones y comportamientos que se manifiestan después de la muerte de una persona significativa, que suelen aparecer a los seis meses de haberse producido el fallecimiento e interfieren en el funcionamiento social, laboral y otras áreas importantes de la persona ${ }^{(7)}$. Estos síntomas están asociados a la aparición de problemas graves de salud, pudiendo generar depresión, hipertensión y problemas cardiacos, abuso de sustancias y drogas, reducción de la calidad de vida ${ }^{(8)}$ y aumento del riesgo de suicidio $^{(9)}$.

A pesar de las propuestas realizadas por parte de los investigadores, en la reciente publicación del DSM-5 ${ }^{(10)}$ el duelo sigue enmarcado dentro del código V62.82, como aquellos problemas adicionales que pueden ser objeto de atención clínica no atribuible a trastorno mental, que a su vez podría ocasionar síntomas similares a la depresión mayor, estrés postraumático, insomnio y anorexia, siendo la evolución crónica e implicando grandes padecimientos y gastos sanitarios. En este caso hay una diferenciación en el DSM-5 con respecto a las anteriores ediciones, y es que el duelo no excluye el diagnóstico de depresión. Si bien el DSM-IV ${ }^{(11)}$ excluía dentro de la depresión a las personas que mostraban dichos síntomas tras la pérdida de ser querido en los dos meses anteriores, el DSM-5 omite esta exclusión.

El duelo sigue siendo un fenómeno complejo que realmente genera muchas cuestiones todavía por dilucidar. Muchos autores comparten la necesidad de aportar datos relevantes que nos ayuden a generar tratamientos bien establecidos, que nos dirijan por el camino más adecuado para poder realizar un buen soporte de las consecuencias que puedan derivarse de su complicación. Centrándonos en este tema, se encuentran muchas publicaciones que muestran un amplio despliegue de diferentes formas de intervención.

En este artículo se va a profundizar en algunos de estos estudios realizados. Concretamente se va a describir los resultados que ha mostrado el reciente Tratamiento de Duelo Complicado de Katherine Shear ${ }^{(12)}$, los correspondientes al grupo de investigaciones que muestran la respuesta dada por los fármacos y los hallazgos que se han aportado desde la terapia cognitivoconductual. Asimismo, se van a presentar datos de algunas de las revisiones sistemáticas que hay en la literatura, las propuestas de los modelos clásicos de intervención del duelo y algunas recomendaciones que se han aportado desde los autores relevantes en el ámbito clínico.

\section{REVISIONES DE LA INVESTIGACIÓN EN TRATAMIENTO DEL DUELO}

Los expertos ponen de manifiesto que la falta de criterios consensuados y medidas sensibles del duelo, dificulta el desarrollo y replicación de las investigaciones, que faciliten conocer cuáles son los tratamientos más efectivos para este tipo de dolor $^{(7)}$.

En las revisiones sistemáticas realizadas de los tratamientos del duelo, se han documentado resultados limitados de diversos 
tipos de psicoterapias. En algunos casos se han llegado a constatar incluso efectos negativos de algunos de los protocolos de actuación, como son los llevados de forma preventiva $^{(13)}$.

En estudios más recientes ${ }^{(14)}$, se muestran resultados más alentadores para este tipo de intervenciones, aunque se continúan cuestionando la validez de los mismos, dado la existencia de las limitaciones metodológicas que presentan.

La literatura científica muestra evidencias de que las intervenciones en duelo normal, pueden interferir en la elaboración del proceso, si se realizan justo después de producirse la pérdida ${ }^{(15,16)}$. Por otra parte, son los tratamientos realizados para duelos complicados o con personas que presentan criterios altos de riesgo, los que muestran respuestas más prometedoras en sus resultados ${ }^{(13-15)}$.

Un reciente metaanálisis ${ }^{(17)}$, aporta una revisión sistemática de los resultados obtenidos a corto plazo y a largo plazo, de las intervenciones preventivas y el tratamiento del duelo complicado.

En esta revisión, se han examinado un total de 14 estudios que han cumplido los criterios de inclusión. Nueve de ellos muestran datos de intervenciones preventivas y cinco para tratamiento de duelo complicado.

Algunos de los hallazgos principales encontrados son:

- Tres de los nueve estudios con intervenciones preventivas ofrecen resultados moderadamente positivos para las manifestaciones del duelo complicado. Dos de ellos cuentan con orientación cognitivo-conductual.

- Por otro lado, de los cinco estudios examinados que realizan tratamiento, cuatro de ellos muestran resultados positivos. Al igual que en las intervenciones preventivas, la orientación de las técnicas aplicadas en esos estudios son cognitivo-conductuales. Estos últimos resultados se reflejan tanto en los análisis realizados a corto plazo y en los seguimientos a largo plazo.

Según los autores, se evidencia a través de este estudio, que las intervenciones preventivas producen o bien efectos positivos o negativos. La falta de resultados de estas intervenciones, puede ser por la posibilidad de que el dolor prolongado no se puede prevenir ni impedir, pero si tratar, tal como se ha demostrado en estudios anteriormente realizados ${ }^{(18)}$. Lo que no significa que se vea mermada la utilidad de este tipo de intervenciones a nivel cualitativo e individual. Además de ello, no se incluyeron en este metaanálisis investigaciones que evaluaran factores de protección del riesgo de duelo complicado, ni se han tenido en cuenta, los beneficios que se tienen cuando son los mismos dolientes los que demanda la ayuda(19).

Los autores de esta investigación señalan las limitaciones metodológicas que se han encontrado en la revisión de los estudios y la dificultad desencadenada por la falta de reconocimiento oficial del diagnóstico de duelo complicado en el DSM.

Como conclusión final plantean que no pueden establecer cuál es el tratamiento más adecuado para duelo complicado. Quizás porque cada forma de dolor es único y genera necesidades diferentes en las personas y que posiblemente los protocolos se debieran adaptar a intervenir de manera individualizada.

\section{PROTOCOLO DE LAS INTERVENCIONES CLÁSICAS. MODELO DE WORDEN}

Como clínicos nos enfrentamos a una difícil tarea a la hora de poder abordar y realizar buenos tratamientos del duelo complicado. Dentro los modelos de trabajo que cuentan con bastante aceptación son las intervenciones por fases y por tareas. Concretamente Worden describió 
cuatro tareas básicas, que consideraba fundamentales a la hora de elaborar este proceso $^{(20)}$ :

a) Aceptación de la realidad de la pérdida.

b) Identificar y expresar sentimientos.

c) Adaptarse a vivir en un mundo en el que el otro ya no está.

d) Facilitar la recolocación emocional del ser querido y seguir viviendo.

A continuación se desarrolla la descripción de estas tareas:

Primera tarea: Aceptar la realidad de la pérdida. Lo que se pretende en estos momentos es intentar entender que la persona que ha fallecido no va a volver, no se va a verla nunca más, ni a poder compartir nada más con ella. Se trata de superar la negación inicial en la que se desenvuelve la persona, que se da para amortiguar el impacto del golpe que supone la realidad a tiempo real y con todas sus consecuencias. Hablar de los rituales funerarios y describir las circunstancias que rodearon a la muerte, invitarlos a visitar el cementerio o el sitio donde se encuentre el difunto, dirigirse a él en pasado, puede ser útil para que la persona acepte la realidad de la pérdida.

Segunda tarea: Ayudar al superviviente a identificar y expresar sus sentimientos. Cuando la persona se empieza a dar cuenta de que todo lo ocurrido es cierto y que no va a cambiar, se intensifican las emociones propias del duelo. El asesoramiento va dirigido a encauzar y expresar las emociones, permitir su expresión y normalizarlas. Para ello puede ser útil escribir lo que siente o hacer uso del dibujo para ejemplificarlo, traer fotos y hablar del fallecido, hacer uso de la técnica de imaginación guiada, reestructurar cognitivamente pensamientos erróneos que pudieran surgir, escribir un pequeño diario o libro de recuerdos que incluyan acontecimientos vividos, historias, fotografías...

Tercera tarea: Adaptarse al día a día, aprender y asumir responsabilidades de la persona fallecida. No suele ser fácil continuar con la vida en los días y meses posteriores a la pérdida, cuanto más tener que encargarse de todo lo que la otra persona asumía. En este momento se enseña a poder resolver problemas y a generar estrategias y herramientas que ayuden a afrontar y responder a las demandas, dándole soluciones adecuadas a las mismas.

Cuarta tarea: Recolocar emocionalmente al fallecido y seguir viviendo. El superar este proceso implica: no olvidar a la persona fallecida sino recordarla sin dolor, empezar nuevas relaciones, establecer nuevos vínculos encontrando un lugar adecuado a la persona que se ha perdido, un lugar emocional transformado, un espacio que no impide ser compartido con los otros ocupados por otras personas y que no impida que la persona esté viviendo el presente y vuelva a vivir.

\section{TRATAMIENTO DE DUELO COMPLICADO, MARCO TEÓRICO}

El tratamiento de duelo complicado planteado por Katherine Shear (21), está indicado para aquellas personas que después de haber perdido a un ser querido, manifiestan dificultades para integrar su nueva realidad en sus vidas.

Está enmarcado dentro de la perspectiva planteada por la teoría del apego y hace uso de técnicas utilizadas en la terapia interpersonal y en la terapia cognitivo conductual e intervención motivacional.

Los planteamientos teóricos en los que se asienta su tratamiento provienen según los propuestas de la teoría de Bowlby $(1985)^{(22)}$. Dentro de esta propuesta se contempla la existencia, a nivel sistema nervioso central, del circuito cognitivoafectivo de apego denominado "working model" y evidencia que la motivación de acercamiento a las figura de apego y de seguridad, se hacía de manera neurobiológicamente instintiva. 
El "working model", incluye elementos de información explícita e implícita, que en nuestra memoria hace diferir el tipo de información que incluye y en la manera en la que asimilamos nueva información. Cuando perdemos a un ser querido, nuestra memoria narrativa incorpora los hechos y acontecimientos ocurridos. Mientras que nuestra memoria semántica e implícita tarda en desarrollar nuevas reglas en las que nuestro ser querido no está presente.

Los síntomas agudos del duelo, en un principio, producen cambios en las emociones, cogniciones y comportamientos de los dolientes. El sistema de apego se interrumpe, se generan sentimientos iniciales de incredulidad, pensamientos intrusivos y comportamientos disruptivos, hasta que poco a poco, se va realizando la integración y dotando sentido a la pérdida de la figura de apego. Es entonces cuando se activa el sistema de nuevo y se restaura el sentido de nuestra vida sin esa persona. Cuando las personas, con el tiempo, no logran redirigir su camino sin la persona fallecida, evitan la expresión de los sentimientos y los pensamientos que generan ansiedad de separación, amargura, quejas o sentimientos de culpa por lo ocurrido y relacionado con la muerte, es cuando pueden aparecer las complicaciones del duelo.

Al narrar y recordar la muerte del ser querido, se puede facilitar el aprendizaje de la memoria implícita. Pasar tiempo pensando en el presente y en el mundo sin la persona querida, facilita la revisión del modelo de trabajo. Esa revisión, con el tiempo, se traducirá en la capacidad de reconstrucción e integración de lo sucedido, del bienestar y sentido de vida.

\section{TRATAMIENTO DEL DUELO COMPLICADO (TDC)}

El tratamiento de duelo complicado, de Katherine Shear, ha mostrado apoyo empírico en un estudio en el que se comparó con la terapia interpersonal ${ }^{(12)}$. En las dos condiciones de intervención, la terapia se administraba en 16 sesiones de 45 y 60 minutos de duración. El diseño de la intervención estaba programada en torno a un periodo de 4 meses.

Los pilares y herramientas fundamentales de la intervención se manifiestan en los siguientes objetivos ${ }^{(21)}$ :

- Información sobre el duelo, su complicación y la terapia de duelo complicado.

- El uso de registros diarios acerca de las emociones y vivencias de dolor relacionadas con la persona fallecida.

- Implicarse y crear otros significados

- Facilitar un funcionamiento óptimo en las relaciones sociales

- Trabajo en los proyectos y objetivos personales

- Recordar y hablar del momento del faIlecimiento de nuestro ser querido y de las implicaciones y consecuencias que ha tenido en nuestra vida.

- Visitar lugares y realizar actividades que se han ido evitando.

- Trabajar a través de recuerdos, fotografías y conversaciones que se imaginan con la persona fallecida.

Como se ha señalado anteriormente, las técnicas psicológicas, que usa esta terapia, proviene de los modelos de trabajo de la psicoterapia interpersonal, la terapia cognitiva conductual usada en el tratamiento del Trastorno de estrés postraumático y estrategias que se usan en intervenciones motivacionales.

La metodología del tratamiento de duelo complicado se estructura en tres bloques principales, en donde las tareas se planifican según los objetivos de cada fase, se trabaja durante las sesiones y se mandan tareas para la siguiente semana.

El primer bloque: La fase introductoria del tratamiento. Se desarrolla durante las tres primeras sesiones del mismo. El principal objetivo se centra, en obtener una historia clínica completa de las relaciones 
interpersonales que tienen el paciente con su familia y se registran qué circunstancias rodearon a la muerte de su ser querido. Asimismo, se recoge información de cómo se vivieron otras pérdidas anteriores o si existen otras en la actualidad. Para finalizar, se explora cómo son las relaciones sociales que tiene en su vida actualmente, las situaciones de estrés que están presentes y los recursos de afrontamiento que tiene el paciente. Este bloque se completa aportando información sobre cómo va a ser la terapia y el modelo de tratamiento a seguir.

El segundo boque: Fase intermedia del tratamiento. Se desarrolla desde la cuarta hasta la novena sesión. En este momento el paciente realiza ejercicios, tanto en las sesiones como en casa, para facilitar una integración de lo que le ha supuesto la pérdida y se trabajan las emociones que desencadenan el recuerdo de la muerte de su ser querido. El objetivo principal es proporcionar una visión racional de la experiencia que se ha vivido, que le permitan convivir también con la continuación de sus aspiraciones personales, su capacidad de disfrute y el sentido de su vida. Este momento del tratamiento es el trabajo central de la terapia. Todas las técnicas van encaminadas a dotar al paciente de la capacidad de revivir lo ocurrido, ser capaces de expresar su dolor y de exponerse a todos aquellas situaciones y lugares que normalmente tienden a evitar, ante la dificultad que sienten de manejar sus emociones. Otro de los aspectos en los que se centra la intervención es en recordar las características y situaciones positivas relacionadas con la persona querida. Para finalizar este módulo, se realiza una síntesis y valoración de los objetivos importantes que tenían en su vida, que no se compartían con su ser querido y se integra la capacidad de disfrutar y sentir satisfacción.

Tercer bloque. Fase final del tratamiento. El terapeuta y el paciente revisan los avances alcanzados y planifican qué objetivos de trabajo necesitan fortalecer para completar e integrar el tratamiento. A partir de aquí, la terapia puede estar relacionada con el cierre de la pérdida y la cercanía de su ser querido o con el manejo de otras pérdidas. Se puede incitar el fomento de nuevas relaciones interpersonales e incluso hacer redefinición de sus propios roles. Hay pacientes con los que se requiere trabajar los sentimientos que les genera la pérdida de la relación que ha mantenido con el terapeuta.

Las técnicas psicológicas con las que se trabajan son ejercicios de exposición en imaginación y en vivo, relatar y recordar las vivencias relacionadas con la muerte de la persona querida, escuchar sus propios recuerdos y expresar qué les hace sentir. Durante el tratamiento se usan fotografías, objetos personales del fallecido, role-playing y conversaciones con la persona fallecida.

Como anteriormente se ha comentado, para validar los resultados de este tratamiento ${ }^{(23)}$, se realizó un estudio prospectivo con pacientes que presentaban criterios de duelo complicado. Para ello se comparó los resultados de la intervención psicológica realizada en una muestra total de 95 dolientes, que fueron asignados aleatoriamente para que se trabajara a través de la terapia interpersonal (IT) y el tratamiento de duelo complicado (TDC). La mayoría de los pacientes eran mujeres con una media de edad de 46 años. El inicio de la investigación se produjo cuando había transcurrido un mínimo de seis meses desde la pérdida de sus seres queridos. Todos los participantes del estudio fueron evaluados a través del Inventario de duelo complicado. Las puntuaciones obtenidas en este cuestionario fueron a partir de 30. La distribución de la muestra al tratamiento fue de 46 dolientes, con los que se intervino a través de la IT y de 49 personas que recibieron TDC, todos ellos 
presentaban diferentes tipos de pérdidas y pertenecían a diferentes estatus social.

Casi en el $50 \%$ de los participantes del estudio se encontraba comorbilidad con otros trastornos psicopatológicos, concretamente, el $20 \%$ tenía sintomatología compatible con el trastorno depresivo mayor y en un $25 \%$ aproximadamente con el trastorno de estrés postraumático.

Los resultados que se obtuvieron fueron más prometedores para la muestra de personas que se trataron con el TDC, mostrando también una mejoría clínica más rápida en el tiempo. Los análisis se realizaron a través de las puntuaciones obtenidas en el Inventario de depresión de Beck y la Escala de Ajuste social y laboral.

\section{TERAPIA FARMACOLÓGICA PARA EL TRATAMIENTO DEL DUELO}

A partir del estudio de Shear, se examinó el papel que pudiera tener el tratamiento con antidepresivos en combinación con la TCD y la IP ${ }^{(24)}$. Una de las cuestiones que suscitaron mayor interés fue que el 91\% de la muestra que estuvo tomando medicación, tenía una tasa de abandono al tratamiento mucho menor, frente al grupo tratado con la TDC únicamente $(91 \%$ vs $58 \%$ ). En el caso de la IT, no se producían los mismos efectos (70\% vs $77 \%$ ) en los pacientes evaluados. Debido a ello, se plantea la necesidad de realizar estudios prospectivos en donde, se examinen, los efectos del tratamiento con antidepresivos y la psicoterapia, tanto en combinación como de manera aislada.

Previamente a estos hallazgos, se han realizado varias investigaciones para valorar la eficacia de los tratamientos farmacológicos en personas con duelo complicado. Algunas de las investigaciones realizadas con psicofármacos son las siguientes:

- Zygmond et al. ${ }^{(25)}$ realizó en 1998, un estudio abierto con 21 personas que puntuaban más de 20 en el Inventario de duelo complicado. En este caso les administró una dosis media de $30 \mathrm{mg} /$ día de Paroxetina en combinación con psicoterapia centrada en el duelo patológico. De la muestra total del estudio, 15 personas completaron 6 semanas de tratamiento y sólo 13, 16 semanas del mismo. Los resultados obtenidos mostraron mejoría del $48 \%$ y del $51 \%$ de la sintomatología del duelo. Las puntuaciones se midieron en el Inventario de duelo complicado y en el Inventario de Hamilton para la depresión respectivamente.

- Simon et al. ${ }^{(26)}$ en el 2007, realizó un estudio de casos de 4 mujeres con una media de edad de 41,75 años. Se les trató con Escitalopram, en dosis flexibles de 10-20mg/día durante 10 semanas. El tiempo que había trascurrido tras la pérdida era de más de 6 meses. A pesar de que el diagnóstico principal fuera de duelo complicado, el $75 \%$ de las participantes presentaban criterios de trastornos de estrés postraumático relacionados con la pérdida. Un $75 \%$ presentaban además historias previas de trastornos ansiosos y en un $100 \%$ había experimentado a lo largo de su vida algún episodio depresivo mayor (el $50 \%$ en el momento en el que se hizo el estudio y el resto en el pasado). Los resultados indicaron una mejoría del $76 \%$, según las respuestas evaluadas a través del Inventario de duelo complicado.

- Hensley et al. ${ }^{(27)}$ en el 2009, estudió de nuevo los efectos del Escitalopram administrado en dosis flexibles de 10-20mg al día, a 14 personas, que cumplían criterios de depresión mayor y duelo complicado. El tiempo que transcurrió tras la pérdida fue de 6 meses y la duración del estudio de 12 semanas. Los resultados muestran una mejoría de la sintomatología de duelo. Las puntuaciones del $21 \%$ los participantes fueron más normalizadas, en el Inventario de duelo complicado.

Como conclusiones generales y según resultados obtenidos en los diferentes es- 
tudios realizados, se plantea que los ISRS pueden tener ciertos beneficios para el tratamiento de duelo complicado y sintomatología depresiva, y parece ofrecer mejores resultados, cuando se administran concominantes con terapias psicológicas. Por otro lado, hay otro grupo de investigadores que proponen que se necesitaría profundizar, en los sistemas de los neurotransmisores y de los circuitos de estrés, para poder conocer más la biología y farmacoterapia del duelo ${ }^{(28)}$.

\section{ORIENTACIÓN COGNITIVO- CONDUCTUAL}

Para entender los puntos clave de su tratamiento, es necesario describir los mecanismos que subyacen en este proceso y que éste modelo considera que son relevantes en su implicación y desarrollo ${ }^{(29)}$.

Dentro de la conceptualización cognitiva-conductual, se consideran que hay tres variables que son fundamentales en la aparición y mantenimiento de la complicación del duelo:

- Las personas con duelo complicado realizan una mala elaboración e integración de la pérdida en la base de datos de conocimientos autobiográficos.

- Presentan creencias globales y negativas, además de una inadecuada interpretación de las reacciones y manifestaciones que se producen.

- Por último, se desarrollan estrategias de afrontamiento ansioso-depresivas a la hora de abordar lo sucedido.

Hay una serie de variables que se consideran antecedentes y que facilitan cómo se puede desarrollar la interacción de estos tres aspectos fundamentales. A partir de aquí se desencadenan la persistencia del duelo complicado manifestándose la clínica principal y propia del mismo, relacionada con la presencia de ansiedad de separación y el estrés desencadenado de la experiencia traumática. Estas variables son: a) los factores vulnerabilidad personal de los dolientes, b) las características que rodearon al suceso, y c) las características de la pérdida.

El tratamiento que se plantea, desde este marco teórico, se fundamenta en intervenir en los factores anteriormente descritos. La dirección del trabajo se detiene en profundizar:

- La necesidad de realizar un procesamiento adecuado de lo sucedido e integrar la pérdida en la autobiografía existente.

- Identificar y cambiar las creencias e interpretaciones del proceso que sean problemáticas.

- Reemplazar las estrategias ansiosodepresivas de evitación, por otras que proporciones un ajuste más adecuado.

En un principio el desarrollo el tratamiento se centra en describir la conceptualización y formulación de los problemas que tiene el paciente. Se proporciona psicoeducación e información de cómo es el desarrollo del tratamiento. En segundo lugar, se lleva a cabo una fase intermedia de la terapia que consiste en realizar una inversión de los procesos que mantienen la complicación del duelo. En este punto es donde se centra la parte más relevantes del tratamiento. Por último se finaliza la intervención, evaluando cómo ha sido el curso general del mismo y se trabaja en la prevención de recaídas.

\section{Inicio del tratamiento}

Las sesiones de trabajo comienzan con una evaluación exhaustiva de los diferentes componentes del modelo. De manera general se hace un registro de cuáles son las cogniciones, emociones y comportamientos que están presentes en el repertorio del paciente.

Se evalúa la problemática principal del paciente y se formulan hipótesis que des- 
criben los factores que favorecen su mantenimiento y desarrollo de las interacciones que se están dando.

Los autores plantean una serie de estrategias para trabajar las cogniciones y facilitar las claves del registro de cómo se siente y vive la pérdida, y que a su vez, pueden ayudar a esclarecer y dar pistas del caso:

- Se les pregunta si consideran la pérdida como algo reversible o definitivo.

- Los recuerdos que se presentan con carácter intrusivo, proporcionan información acerca del abordaje posterior que se ha de realizar de la pérdida.

- La presencia de eventos estresantes relacionados con la muerte, pueden informarnos si hay un mal procesamiento de lo sucedido.

- Para poder evaluar las creencias que tiene el paciente, se les pide que describan cómo fue la muerte del ser querido y si todo lo acontecido ha podido cambiar su punto de vista sobre sí mismo, su vida y su futuro. En este momento se puede pasar algún cuestionario que evalúe las cogniciones relacionadas con el duelo, como por ejemplo el Cuestionario de cogniciones de duelo ${ }^{(30)}$.

- Con respecto al trabajo centrado en las emociones; la evaluación también debe incluir experiencias anteriores con la pérdida, fortalezas y debilidades que estén presentes en su repertorio de afrontamiento. Las creencias preexistentes sobre el yo y el mundo $y$ las consecuencias que ha tenido vivencia.

Para completar la parte inicial del bloque, se ofrece información de cuáles son las manifestaciones y reacciones propias del duelo, cómo funcionan las emociones, cómo se encauzan y cuáles son los comportamientos habituales. Se les ofrece una perspectiva de cómo transcurre y se elabora el proceso, tratando de proporcionar un marco de compresión general que favorezca la normalización de lo que el paciente está viviendo.

\section{Facilitar la integración de la pérdida en la bases de conocimiento autobiográfico}

En este momento, la intervención se orienta hacia el logro y aproximación de un conocimiento real de lo que la pérdida ha significado en su vida.

El trabajo se centra en que la persona reconozca los sentimientos más dolorosos, los pensamientos y recuerdos vinculados a la pérdida. Se hace hincapié en la problemática que está interfiriendo en la integración de lo sucedido y en la reorganización de su mundo interior y se trata de disminuir las reacciones emocionales más difíciles de manejar para favorecer su ajuste.

Las estrategias van encaminadas principalmente a realizar una exposición en imaginación o en vivo, en la que se revive la historia de lo acontecido y cómo se han ido traduciendo las consecuencias de la muerte en la vida cotidiana del doliente. Se puede poner en marcha las técnicas de inundación usadas para el tratamiento del Trastorno de estrés postraumático de $\mathrm{FOA}^{(31)}$.

La exposición se realizaría de manera graduada para que se vayan rompiendo las creencias globales más negativas, que sostienen la evitación de las emociones que son dolorosas. Todo ello va encaminado a facilitar un tránsito a creencias más realistas que favorezcan el ajuste y adaptación.

\section{Cambio de las creencias negativas y las inadecuadas interpretaciones de las reacciones del duelo}

El terapeuta interviene a través de diálogo socrático, en las creencias que se han ido identificando a lo largo de la terapia. Las interpretaciones catastróficas se 
van trabajando mediante reestructuración cognitiva y experimentos adaptados al repertorio de comportamiento del paciente. Con el objetivo de desmontar las cogniciones erróneas, se van aportando pruebas, de que la validez de las interpretaciones está magnificada y sobrevalorada.

La elaboración de los significados y la conciencia de lo que ha supuesto la pérdida, se acentúan a medida que se van realizando las exposiciones de los recuerdos dolorosos a la vez que se va disminuyendo su evitación.

Para reducir la evitación ansiosa y depresiva:

El papel que realizan las técnicas de exposición, en esta terapia, ocupa un lugar protagonista. Se centran en la identificación de los pensamientos y creencias que conducen a evitar entender la irreversibilidad de la pérdida. Se valoran las consecuencias a corto y largo plazo de estos supuestos.

Para trabajar la ansiedad que genera este abordaje, las exposiciones se nutren además con la prevención de respuesta que se suele usar en el tratamiento de los Trastornos obsesivos-compulsivo.

Para fortalecer el trabajo realizado con las reacciones depresivas, se planifican el incremento de actividades placenteras que estaban presentes en la vida cotidiana del paciente con anterioridad a la pérdida. Todo ello con el objetivo de favorecer que recupere y mejore su estado de ánimo y calidad de vida.

Para finalizar el tratamiento se hace una valoración general del transcurso de la terapia. Para ello, se identifica, si el desarrollo terapéutico se ha ajustado a los objetivos que inicialmente se plantearon, si la mejoría clínica de los pacientes se ha corroborado en la comprobación de las hipótesis que se formularon al principio de la intervención y por último, si la terapia ha favorecido el manejo de las emociones que estaban interfiriendo en la elaboración y encauzamiento del proceso del duelo.

\section{TRATAMIENTO DEL DUELO DESDE EL ÁMBITO CLÍNICO}

\section{Counselling}

Hay autores que consideran el counselling como un marco de intervención idóneo ante el fenómeno del duelo ${ }^{(32)}$. El counselling, se entiende como un proceso interactivo, en el que respetando el principio de autonomía de la persona y a través de la empatía, de la confrontación y de diversas estrategias de comunicación, se acompaña a que las personas tomen las decisiones que sean más adecuadas para sí mismos y su estado emocional ${ }^{(33)}$.

A través de sus principios fundamentales y actitudes básicas: la empatía, la veracidad y la aceptación incondicional, se hincapié en entender y la situación del otro y a su vez favorece que el paciente se sienta comprendido y capaz. Se trabaja desde la autenticidad, la aceptación y respeto a sus valores y se va a tratar de acompañarle y a poner en marcha todas las herramientas terapéuticas que sean necesarias, para facilitar la elaboración del proceso y alivio de su sufrimiento. Para ello, se requiere unos conocimientos técnicos de los aspectos en los que se va a profundizar, y ser conscientes como profesionales de la vinculación y contacto con la vulnerabilidad y dolor con el que vamos a tratar.

Hay una serie de herramientas que están presentes y se tiene en cuenta durante este tipo de intervención que a su vez facilitan la relación con el paciente:

- Habilidades de comunicación.

- Soporte emocional.

- Modelo de solución de problemas.

- Autocontrol.

Todo lo anteriormente planteado son los principios básicos generales en los 
que se sustenta esta herramienta terapéutica de intervención. A través del dialogo deliberativo y soporte emocional ${ }^{(33)}$, se fomenta a que el paciente tome parte en su capacidad de autocuidado, a estimular la búsqueda de soluciones y la toma de decisiones que mejoren su propio bienestar.

\section{APORTACIONES DESDE EL ÁMBITO CLÍNICO}

Para finalizar este artículo, se van a señalar algunos principios terapéuticos y objetivos de intervención, que desde el ámbito clínico han descrito Barreto y Soler ${ }^{(32)}$ como autores referentes en el estudio y abordaje del duelo.

En el inicio de la intervención del duelo plantean una serie de recomendaciones, que pueden mediar en la comprensión y encaminar la dirección y manejo del tratamiento. En un primer momento, indican que en este tipo de soporte, es adecuado hacer entender al paciente el tipo de relación que se va a mantener con el terapeuta, planificando el número y el objetivo terapéutico de las sesiones; además de ello, parten de la relevancia en aclarar e informar en qué consiste elaborar la pérdida de su ser querido, haciendo hincapié en que superar no significa olvidar; se explica la transición que conlleva los momentos iniciales en los que se vive en el recuerdo constante de lo sucedido, hacia recolocar al fallecido en otro espacio diferente compatible con su vida, en un presente contemplando sus proyecciones futuras. Consideran hacer un buen uso de las estrategias de comunicación no verbal en el soporte e introducir y facilitar en la intervención la expresión emociones positivas.

De manera descriptiva plantean una serie de objetivos según se intervenga de forma preventiva, se realice una terapia más especializada o se profundice en sesiones de mantenimiento.

Cuando se trata de realizar una intervención preventiva antes del fallecimien- to del familiar, se incide en fortalecer las habilidades de autocuidado y autoeficacia del cuidador, fomentando la solución de posibles incidencias y mediando en la conciliación de las diferencias y asuntos que pudieran estar pendientes con el enfermo, que a su vez, pudieran interferir en la despedida de su ser querido. Facilitar la despedida cuando sea posible.

Después de producirse la pérdida, se plantea favorecer la adaptación al duelo y prevención de la complicación del proceso. La línea de trabajo va dirigida a proporcionar información de las reacciones naturales que suelen manifestarse, relatar lo sucedido facilitando la expresión y el encauzamiento de emociones de manera normalizada. Fomentar las estrategias propias del paciente y dotar de aquellas que le puedan servir para afrontar y adaptarse a las demandas del día a día, que le ayuden a manejar sus necesidades y a ocuparse de sus problemas y preocupaciones. Facilitar la entrada y soporte de su entorno social cercano. Mantener las habilidades de autocuido personal.

En el caso de tratamientos más especializadas, los objetivos de la intervención se derivan de una evaluación completa, de las necesidades y problemática que tiene el paciente para establecer la dirección de la terapia. Principalmente las actuaciones van encaminadas a fomentar la realidad de lo sucedido y a lograr encontrar una posición de su ser querido en el presente del paciente, que le permita seguir adelante. Se valoran además las consecuencias que ha tenido la pérdida, se restablece su identidad favoreciendo los cambios que ha supuesto en su persona. Se abordan aquellos pensamientos que pudieran interferir en el manejo de las emociones que interrumpan su encauce y la elaboración del proceso. Asimismo, puede ser necesario intervenir en la forma de gestionar sus relaciones sociales, restablecerlas o iniciar otras nuevas. Se recomienda además ser cautelosos en la prescripción farmacológica. 
Por último cuando se realizan intervenciones de mantenimiento, los objetivos van dirigidos a mejorar su calidad de vida $y$ bienestar personal.

Las técnicas psicológicas que se van a utilizar para poder llevar a la práctica estos objetivos pertenecen a diferentes orientaciones psicológicas:

- Condicionamiento clásico y operante, a través de programas de contingencia aplicado para aumentar las satisfacciones del doliente que pudieran equilibrar y balancearse con las vivencia de emociones más dolorosas y que le producen malestar. Fomentar el aprendizaje de nuevos repertorios de conducta. Hacer uso del control estimular para mediar en la aparición de conductas disfuncionales. Se puede aplicar desensibilicación sistemática y la exposición en imaginación y/o en vivo, como acercamiento hacia aquellos sucesos que tienden evitarse $y$ facilitar la expresión de emociones.

- Restructuración cognitiva y ensayos conductuales. Principalmente para trabajar en todas aquellas creencias disfuncionales que puedan interferir en el curso adecuado del proceso, que favorezcan la reconstrucción de nuevos significados y la adaptación a los cambios que le ha supuesto la pérdida.

- Constructivismo, en donde se aplican la escritura de biografías, dibujos e imágenes, cartas que no se envían, galerías fotográficas, historias e imágenes metafóricas, libros de recuerdo..., con lo que se pretende estimular la aparición de un nuevo sentido después de la pérdida y de las creencias que se tienen de la vida.

- Resolución de problemas. Aplicando el protocolo y los pasos de la técnica en sí misma, para favorecer la gestión de las incidencias que pudieran presentarse en el día a día.

- Entrenamiento en habilidades sociales y asertividad. Con el objetivo de poder facilitar la comunicación con sus seres queridos y la expresión de sus necesidades, además de experimentar el apoyo de las personas que son significativas para el doliente.

- Exposición repetida y gradual a los estímulos evitados. En este caso habría que adaptar la intervención según si el doliente evita exponerse a determinadas situaciones, dado el dolor tan importante que manifiestan a la hora de vivir la experiencia, o por el contrario, el evitar separarse de situaciones o rituales que le anclan en el recuerdo y le impide no progresar o no vivir la realidad de lo sucedido.

- Realizar rituales de despedida o aplicar otras técnicas como pueden ser la visualización, juegos de rol, autoinstrucciones positivas, parada de pensamiento y la ventilación de emociones.

\section{CONCLUSIONES}

A lo largo de estos últimos años, se están realizando muchos estudios que exploran las diferentes líneas de intervención del duelo. Hoy en día, es difícil responder o establecer conclusiones sobre cual es tratamiento que pudiera proporcionar más beneficios o ser más eficaz. A pesar de ello, todo el esfuerzo invertido en las investigaciones desarrolladas en este campo, si que aportan orientación y guían las direcciones que se pueden tomar.

En la literatura se han documentado en líneas generales, como los autores descartan realizar cualquier tipo de intervención rutinaria con las personas que están viviendo un duelo normal. Se considera que cualquier actuación que se realice en estos casos, pueden interferir su curso natural y dificultar el desarrollo de las estrategias propias que los dolientes, ponen en marcha para afrontar y elaborar el proceso. Por el contrario, los tratamientos para personas con criterios de alto riesgo y las intervenciones especializadas, cuando el duelo se 
ha complicado, han mostrado resultados más alentadores que se constatan aun más, en los casos en los que son los mismos pacientes los que demandan la atención.

Dentro de este marco de referencia, es difícil encontrar programas específicos que se hayan diseñado para tratar el duelo. Según los estudios realizados, la orientación que muestra ser más eficaz es la que lo aborda desde la terapia cognitivo-conductual. El counselling, a su vez, ha proporcionado resultados positivos en grupos de dolientes con alto riesgo y con manifestaciones psicopatológicas ${ }^{(32)}$.

En este ámbito, el Tratamiento del duelo complicado, propuesto por Katherine Shear, ha mostrado más beneficios que la intervención realizada con terapia interpersonal. Boelen et al. ${ }^{(34)}$ examinaron los resultados que ofrecieron las técnicas de exposición, la reestructuración cognitiva y la terapia de apoyo centrada en la solución de problemas. En su estudio realizaron tres grupos con distintas combinaciones y condiciones de intervención. Los análisis de datos mostraron mejores resultados para los grupos que se trataron con técnicas de exposición y reestructuración cognitiva, que los tratados con terapia de apoyo centrada en la solución de problemas. Asimismo, en la reciente revisión que realiza Wittkouk, van Autreve, de Jaegere, Portzky y van Heeringen de esta misma investigación, refiere que el protocolo que se ha realizado en cada grupo de intervención, ofrece mejorías clínicas significativas en comparación al grupo control y que estos resultados se mantienen en los seguimientos posteriores realizados con los dolientes.

Estas aportaciones están estimulando el desarrollo de estudios en esta línea, ante la necesidad de contar con investigaciones bien establecidas y diseños prospectivos, que permitan aportar más conocimientos y esclarecer las direcciones que los profesionales puedan tomar, para ofrecer el soporte, alivio y el acompañamiento más adecuado, de las personas que están viviendo este proceso y dolor.

\section{REFERENCIAS BIBLIOGRÁFICAS}

1. Barbero J. La muerte de un ser querido. Duelo y adaptación en las personas mayores. Madrid, Portal Mayores, Informes Portal Mayores, $n^{\circ}$ 53. Lecciones de Gerontología, III [Fecha de publicación: 09/06/2006]. [Acceso 13 de septiembre de 2013].Disponible en: http://www.imsersomayores.csic.es/documentos/documentos/ barbero-muerte 01.pdf

2. Bayés R. Psicología del sufrimiento y de la muerte. Barcelona: Ediciones Martínez Roca, 2001.

3. Prigerson HG, Maciejewski PK, Reynold CF, Bierhals AJ, Newsom JT, Fasiczka A, et al. Inventory of Complicated Grief: A scale to measure maladaptive symptoms of loss. Psychiatry Res 1995;59:65-79. Doi: 10.1016/0165-1781(95)02757-2.

4. Prigerson HG, Horowitz MJ, Jacobos SC, Parkes CM, Aslan M, Goodkin K, et al. Prolonged grief disorder proposed for DSM V and ICD-11. PLoS Med 2009;6. Doi: 10.1371/journal.pmed.1000121

5. Jacobs S, Prigerson H. Psychotherapy of traumatic grief: a review of evidence for psychotherapeutic treatments. Death Stud 2000;24:479-95.

6. Lichtenthal WG, Cruess DG, Prigerson HG. A case establishing complicated grief as a distinct mental disorder in DSM-V. Clin Psychol Rev 2004; 24: 237-62.

7. Prigerson H, Vanderwerker LC, Maciejewski PK. A case for inclusion for Grief Prolonged in DSM-V. In: Stroebe M, Hansson R, Schut $\mathrm{H}$, Wolfgang $\mathrm{H}$, editors. Handbook of bereavement research and practice: $21^{\text {st }}$ Century Perspectives, Washington DC: American Psychological Association, 2008; p. 165-186.

8. Boelen PA, Prigerson, HG. The influence of symptoms of prolonged grief disorder, 
depression, and anxiety on quality of life among bereaved adults: A prospective study. Eur Arch Psychiatr Clin Neurosci 2007;257:444-52. Doi: 10.1007/s00406007-0744-0

9. Latham A, Prigerson HP. Suicidality and bereavement: Complicated grief as psychiatric disorder presenting greatest risk for suicidality. Suicide Life Threat Behav 2004;34 (Suppl 4):350-62. Doi: 10.1521/ suli.34.4.350.53737

10. American Psychiatric Association. Diagnostic and statistical manual of mental disorders fifth edition DSM-5. Washington DC: American Psychiatric Association, 2013.

11. American Psychiatric Association. DSMIV- TR. Manual diagnóstico y estadístico de los trastornos mentales. Texto revisado. Barcelona. 2001.

12. Shear K, Frank E, Houtck PR, Reynold CF. Treatment of complicated grief: A randomized crontroled trial. JAMA, 2005;293:2601-8.

13. Currier J, Neimeyer R, Berman JS. The effectiveness of psychotherapeutic interventions for bereaved persons: A comprehensive quantitative review. Psychol Bull 2008;134:648-61. Doi: 10.1037/00332909.134.5.648.

14. Shut H, Stroebe M. Interventions to enhance adaptation to bereavement interventions: Determining who benefits. J Pall Med 2005;8:140-7. Doi:10.1089/ jpm.2005.8.s-140.

15. Neymeyer RA.Searching for the meaning of meaning: Grief therapy and the process of reconstruccion. Death Stud 2000; 24:541-58.

16. Schut H, Stroebe M, van den Bout J, Terheggen $M$. The efficacy of bereavement interventions: Determining who benefits. In: Stroebe M, Hansson R, Stroebe W, Schut $H$, Terheggen $M$, editors. Handbook of bereavement research: Consequences, coping, and care. Washington DC: American Psychological Association, 2001.p.705-7.
17. Wittouck $C$, van Autreve $S$, de Jaegere $E$, Portzky G, van Heeringen K. The prevention and treatment of complicated grief: A meta-analysis. Clin Psychol Rev 2011;31:6978. Doi: 0.1016/j.cpr.2010.09.005

18 Neimeyer RA, Currier JM. Grief therapy: evidence of efficacy and emerging directions. Current Direc Psychol Sci 2009;18:352-6. Doi:10.1111/j.14678721.2009.01666.x

19. Kang KS, Yoo Y. Effects of bereavement intervention program in middle-aged widows in Korea. Arch Psychiatr Nurs 2007;21:132-40.

20. Worden JW. El tratamiento del duelo: asesoramiento psicológico y terapia. Barcelona: Paidós, 2004.

21. Shear K. Complicated grief treatment: The theory, practice and outcomes. Bereave Care 2010;29 (Suppl 3):10-4. Doi:10.108 0/02682621.2010.522373.

22. Bowlby Y J. El apego y la pérdida. Barcelona: Editorial Paidós, 1985.

23. Simon NM, Shear MK, Fagiolini A, Frank E, Zalta A, Thompson E, et al. The impact of concurrent naturalistic pharmacotherapy on psychotherapy of complicated grief. Psychiatry Res 2008; 159 (Suppl 1-2):31-6. Doi:10.1016/j.psychres.2007.05.011.

24. Mark D, Miller MD. Complicated grief in late life. Dialogues Clin Neurosci 2012;14 (Suppl 2):195-202.

25. Zygmont $M$, Prigerson HG, Houck PR, Zygmont M, Prigerson HG, Houck PR, et al. et al. A post hoc comparison of paroxetine and nortriptyline for symptoms of traumatic grief. J Clin Psychiatry 1998;59:241-5.

26. Simon NM, Thompson EH, Pollack $\mathrm{MH}$, Shear MK. Complicated grief: A case series using escitalopram. Am J Psychiatry 2007;164:1760-1. Doi: 10.1176/appi. ajp.2007.07050800

27. Hensley PL, Slonimski CK, Uhlenhuth EH, Clayton PJ. Escitalopram: An open-label study of bereavement-related depression 
and grief. J Affect Disord 2009; 113:1429. Doi: 10.1016/j.jad.2008.05.016.

28. Bui E, Nadal-Vicens M,Simon N. Pharmacological approaches to the treatment of complicated grief: Rationale and brief review of the literature. Dialogues Clin Neurosci 2012;14 (Suppl 2):149-57.

29. Boelen PA, Hout MA, Bout J. A CognitiveBehavioural Conceptualization of Complicated Grief. Clin Psychol Sci Prac 2006; 13 (Suppl 2):109-28. Doi: 10.1111/j.14682850.2006.00013.x.

30. Boelen PA, Lensvelt-Mulders G J. Psychometric properties of the Grief Cognitions Questionnaire (GCQ). ). J Psychopathol Behav Assess 2005;27:291-303. Doi: 10.1007/ s10862-005-2409-5.
31. Foa E, Rothbaum BO. Treating the trauma of rape. Cognitive-behavioral therapy for PTSD. New York: The Guilford Press, 1998.

32. Barreto P, Soler MC. Muerte y duelo. Madrid: Síntesis, 2007.

33. Arranz P, Barbero J, Barreto P, Bayés R. Intervención emocional en cuidados paliativos. Modelo y protocolos. Barcelona: Ariel, 2003.

34. Boelen PA, de keijer J, van de Hout MA, van den Bout J. Treatment of complicated grief: A comparison between cognitive-behavioral therapy and supportive counseling. J Consult Clin Psicol 2007;46:370-80. Doi: 10.1037/0022006X.75.2.277 